\title{
COLLODION BABY- A CASE REPORT AND REVIEW OF LITERATURES
}

\author{
MOLLAH MAH ${ }^{1}$, MAY M ${ }^{2}$, BANERJEE $\mathrm{M}^{\mathrm{C}}, \mathrm{MSR}^{\mathrm{D}}$, KHAN TH${ }^{\mathrm{E}}, \mathrm{RAHMAN} \mathrm{H^{ \textrm {F } }}$
}

\begin{abstract}
A four day old female baby, only issue of a nonconsanguinous parent was admitted with abnormal shiny skin and respiratory distress and who finally diagnosed as Collodion baby. After giving appropriate management the baby was improved and discharged home. The case is reported here for further documentation.
\end{abstract}

J Dhaka Med Coll. 2011; 20(2) : 213-217.

\section{Introduction}

Ichthyosis is characterized by persistently dry, thickened, rough, fish scale skin. There are at least 20 varieties of Ichthyosis, including inherited and acquired forms ${ }^{1}$. The word ichthyosis comes from the Ancient Greek (ichthys), meaning "fish." lamellar ichthyosis (LI) Often called collodion baby is one of a variety of ichthyoses and occur as an isolated and self-limiting condition. Collodion baby is inherited in an autosomal recessive manner and about $10 \%$ of the cases transmitted as an autosomal dominant trait. Sex linked ichthyosis never begins with a Collodion baby syndrome ${ }^{3}$.

\section{The case report}

A 4 days old female baby appropriate-forgestational-age only issue of nonconsanguinous Parents born at local clinic by lower uterine cesarean section (LUCS) due to leaking membrane for $12 \mathrm{hrs}$ and Baby was admitted at Dhaka Medical College Hospital at Special care baby unit on 4 days of age on $4^{\text {th }}$ February 2010, presented as abnormal appearance with respiratory distress. Mother had a history of gestational diabetes Mellitus and she were on dietary management. On examination the baby weighed 2500grams, with a length of $49 \mathrm{~cm}$ and had head circumference of $35 \mathrm{~cm}$. There was everted lower eyelid (ectropion), absence of eyelashes and eye brows; low set, small malformed ears mouth was fishlike (eclabium) and joint stiffness. The skin was semitransparent, parchment like feel with varying degrees of fissures at groin and axilla. The skin at places looked to be yellowish brown glistening and film like membrane resembling scales of fish .Systemic examination did not reveal anything abnormal. The neonatal reflexes could not be adequately elicited due to eclabium and joint stiffness.

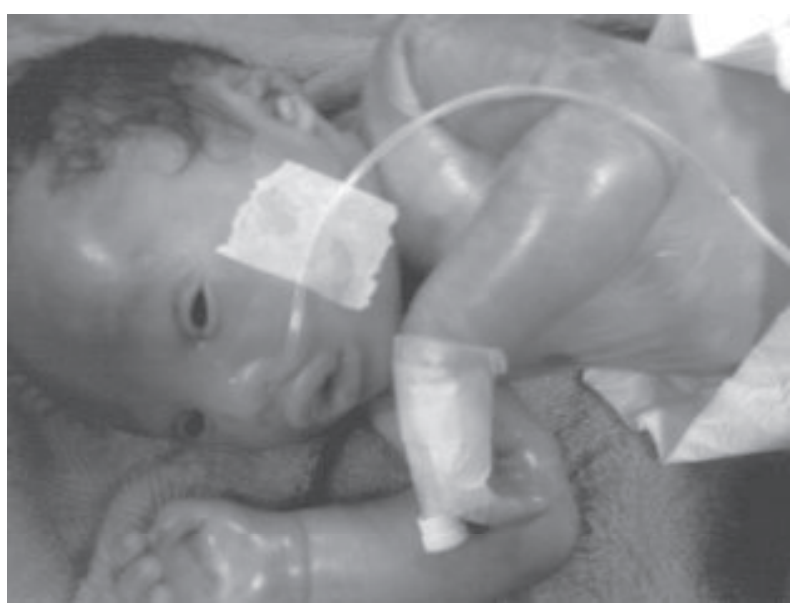

a. Dr. Md Abid Hossain Mollah, Professor \& Head of the Department of Neonatology, Dhaka Medical College \& Hospital

b. Dr. May May, Honorary Medical Officer, Pediatric, Dhaka Medical College \& Hospital

c. Dr. Monisha Banerjee, Assistant Prof. of the Department of Neonatology, Dhaka Medical College \& Hospital

d. Dr. Shishir Ranjan Das, Assistant Prof. of the department of Neonatology, Dhaka Medical College \& Hospital

e. Dr. Taffazzol Hossain Khan, Assistant Prof. of the Department of Neonatology, Dhaka Medical College \& Hospital

f. Habibur Rahman, Assistant register, department of Neonatology, Dhaka Medical College \& Hospital

Correspondence : Dr. Md Abid Hossain Mollah, Professor \& Head of the Department of Neonatology, Dhaka

Medical College \& Hospital 


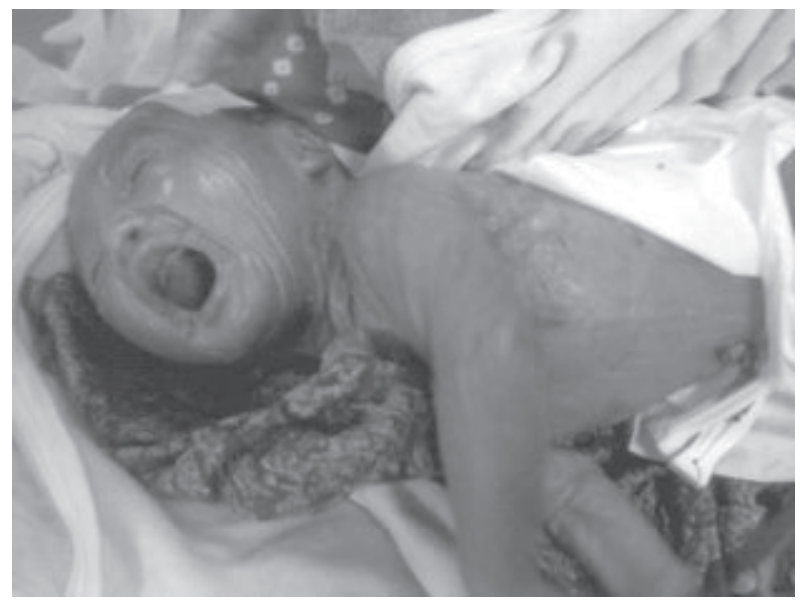

The infant was placed in a humidified isolette after initial stabilization. A 10\%DA was started at a rate of $80 \mathrm{ml} / \mathrm{kg}$ per day. Prophylactic antibiotics consisted of ceftazidime and amikacin. Consultations with Dermatology, ophthalmology for ectropion was obtained. The baby was monitored regarding the temperature, hydration status and electrolyte balance. Bland lubricants were applied to the skin. The skin was kept lubricated to keep the peeling skin soft to facilitate desquamation.(Oilatum) liquid paraffin $63.4 \%$,(Lacticare) 5\% lactic acid, á hydroxy acid was applied twice daily to the area, $\&$ (tobirex) Tobramycin eye drops was applied to the eyes. The infant was started on oral feedings on 4th day of life. Blood was drawn for electrolytes in which $\mathrm{Na}$ level was $159 \mathrm{mmol} / 1$, $\mathrm{K}$ was $5.7 \mathrm{mm01} / \mathrm{L}$. Antibiotics were stopped after 7 days \&the infant was then discharged from the hospital on day 8th of life after skin improvement. The grandparents were instructed to give short baths with water only once a day \& apply liquid paraffin $63.4 \%($ Oilatum) twice daily. Follow up in dermatology, ophthalmology \& otolaryngology as an outpatient was recommended.

\section{Discussion}

Collodion baby is the name given to a baby who is born encased in a skin that resembles a yellow, tight and shiny film or dried Collodion (sausage skin) ${ }^{4}$. The frequency of Collodion baby is very low. It is estimated that there are 1:300,000 cases of newborns in the worldwide ${ }^{5}$. These babies are often premature. The Collodion membrane undergoes desquamation or peeling, which is usually complete by 2 to 3 weeks of life. This reveals the underlying skin disorder The Collodion membrane is due to abnormal desquamation (a peeling process). It is due to mutation of certain genes and is usually an autosomal recessive, congenital ichthyosis (scaly skin condition). However 10\% of Collodion babies have normal underlying skin - a mild presentation known as 'selfhealing' Collodion baby ${ }^{4}$.

Patients with lamellar ichthyosis have accelerated epidermal turnover with proliferative hyperkeratosis, in contrast to retention hyperkeratosis. This involves a mutation in the gene for transglutaminase 1 (TGM1). The transglutaminase 1 enzyme is involved in the formation of the cornified cell envelope. The formation of the cornified cell envelope is an essential scaffold upon which normal intercellular lipid layer formation in the stratum corneum occurs. Thus, mutations in the TGM1 secondarily cause defects in the intercellular lipid layers in the stratum corneum, leading to defective barrier function of the stratum corneum and to the ichthyotic phenotype seen in lamellar ichthyosis patients and in transglutaminase 1 knockout mice ${ }^{5}$. How much a defective cornified cell envelope alone contributes to the barrier abnormality in ichthyoses remains unclear ${ }^{6}$, the pathogenesis of molecular mechanisms apparently lead to an epidermal cornification disorder, keratinocyte protein and lipid metabolism defects resulting from autosomal recessive genetic mutations have also been notified as important cofactors.

Common symptoms includes

- Premature birth

- Tight yellow shiny film on skin

- Infection

- Feeding problems

- Breathing problems

- Everted lower eyelid

- Difficulty regulating temperature

- Dehydration

- Everted lips

- Scaly skin

- Peeling skin ${ }^{7}$ 
The babies are usually covered at birth by a thick taut membrane resembling oiled parchment or Collodion, which is subsequently shed, often resulting in ectropion and eclabium. Within few hours this membrane dries, cracks and peels off but may reform several times. Infrequently, an affected infant has normal skin after the membrane is shed. Affected neonates have ectropion, flattening of the ears and nose, fixation of the lips in an O- shaped configuration. The case discussed in the text also has similar features. Hair may be absent or may perforate the horny covering. The membrane cracks with initial respiratory efforts and shortly after birth, begins to desquamate in large sheets. Complete shedding may take several weeks, and a new membrane may occasionally form in localized areas. The Collodion membrane is composed of greatly thickened stratum corneum that has been saturated with water. As the water content evaporates in extra uterine life, large fissures appear in the membrane and the membrane is shed, revealing red skin underneath $^{8-11}$.

The taut membrane may impair respiration and sucking, sometimes leaving fissures with impairment of barrier to infection and water loss leading to difficulties in thermal regulation and hypernatremic dehydration. Ectropion may result in the inability to fully close the eyelids and can cause exposure keratitis. Neonatal morbidity and deaths may be due to cutaneous infection, aspiration (squamous material) and hypothermia. The outcome is uncertain. The Collodion babies may be born with a Collodion membrane covering the entire skin surface just like armor. Particularly, tight membrane on limbs may lead to constriction and loss of function $(12,13)$. The presence of Collodion membrane doesn't necessarily predict that the baby will develop ichthyosis and spontaneous healing may occur.

The skin biopsies from such babies on first and 15 th day of life when examined in microscope, features observed were different from those known to occur in Collodion babies evolving into lamellar ichthyosis and may contribute to a more precise, early diagnosis and prognosis of this heterogenous neonatal entity $(14,15)$

Fetal skin biopsy at approximately 20-22 weeks gestation has been accomplished in lamellar ichthyosis, congenital ichthyosiform erythroderma, epidermolytic hyperkeratosis and Sjogren-Larsson syndrome. In Harlequin ichthyosis prenatal diagnosis has been successfully performed by fetal skin biopsy and ultrasonography. This can be done with chorionic villous sampling ${ }^{12}$.

With light microscopic examination of the skin specimens of a newborn baby with a Collodion membrane in the early periods an eosinophilic, PAS positive stratum corneum accompanied by hyperkeratosis can be observed. However, the epidermis is weakened due to the thinning of the granular layer. But electron microscopic examination reveals dense intracytoplasmic granules and convoluted corneocytes that can be seen in the upper portion of stratum corneum. Lamellar bodies are numerous but intercellular space and the desmosomes are well preserved. The thinned granular layer is structurally normal. Skin biopsy of Collodion membrane is usually not diagnostic. Most Collodion babies do have a form of ichthyosis and majority of them develop features of lamellars, bullous \& X-linked ichthyosis, Netherton's syndrome or Gauchers disease ${ }^{16}$.

Treatment with a high - humidity environment and application of non occlusive lubricants may facilitate shedding of membrane. Long baths using bath oils or application of lubricants before drying can prolong the hydration and softening. Keratolyic agents (creams and lotions containing urea, salicylic acid and alpha hydroxyl acids like lactic and glycolic acids) are used to enhance corneocytes disadhesion. Topical retinoid or vitamin D preparations may also be effective but can be irritate in some patients. Decreased sweating and heat intolerance should be explained to the parents and they should be made aware of signs of heat intolerance such as flushing and lethargy. Avoiding hot environments and carrying spray bottles with water to moisten the skin and cool through evaporation. A spontaneously healing Collodion baby has been reported in 10-25\% cases ${ }^{14}$. 
Stabilization Care of the Collodion baby

Impaired skin integrity 18

Goal: Prevent further impairment of skin barrier function \& maintain skin integrity to prevent sepsis.

Risk for altered body temperature

Goal: Minimize TEWL \& heat loss while maintaining skin temperature between $36.5^{\circ} \mathrm{c} \& 37^{\circ} \mathrm{C}$.

Altered nutrition:

Less than body requirements

Goal: Provide the most appropriate

nutritional management based on the infants tolerance.

Sleep pattern disturbance:

Goal: Promote normal infant sleep/wake states
1. Assess open skin lesions.

2. Minimal handling, vital signs per routine.

3. Use standard precautions with handing.

1. Avoid radiant heat.

2. Isolette with high humidity.

3. Pre-warm all direct skin contact linen used to absorb weeping.

1. Evaluate enteral feeding ability.

2. Closely monitor hydration: I\&O, moist mucous membranes, wt

1. Position for comfort with flexed positioning or by holding..
This condition is an autosomal recessive genetic disorder ${ }^{17}$, which means the defective gene is located on an autosome, and both parents must carry one copy of the defective gene in order to have a child born with the disorder. Carriers of a recessive gene usually do not show any signs or symptoms of the disorder

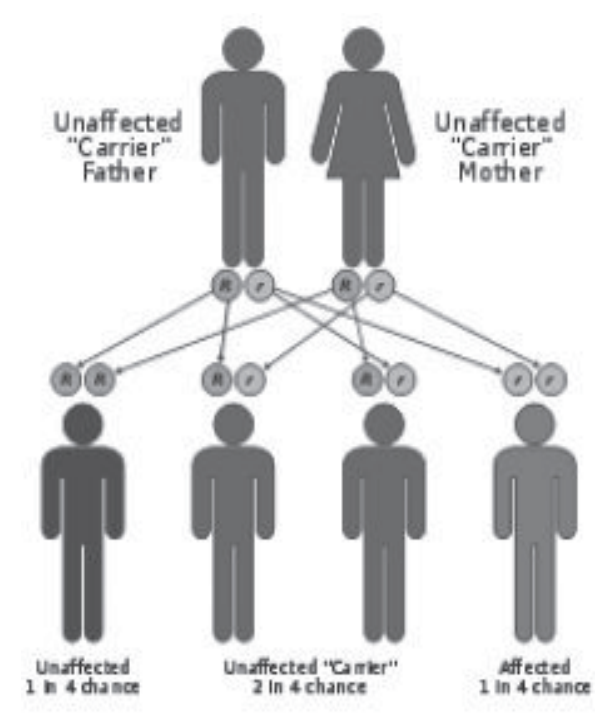

Ichthyosis lamellaris has an autosomal recessive pattern of inheritance
Approximately 1:225 individuals are a carrier. The risk to future pregnancies is $25 \%$. Unaffected siblings of an affected individual have a $2 / 3$ risk of being a disease gene carrier 15. Missense mutations spice - site mutations and deletions occur throughout the gene. Transglutaminase -1 (TGM1) is the only gene identified to date to be mutated in patients with lamellar ichthyosis

\section{Conclusion}

Early recognition of this clinical entity and early institution of appropriate therapy can definitely reduce morbidity and mortality in neonates. Because this is a rare disease it is indispensable to have very clear and precise information on the steps to follow and the complications that may arise.

\section{References}

1. Created 2003. Last updated 05 Jun 2010. (C) 2011 NZDSI.

2. Ichthyosis, John Hopkins Health Information Library

3. Morelli JG. Disorders of keratinazation. In: Behrman RE, Klieg man RM, Jenson HB (eds) Nelson Textbook Of Pediatrics, 18th edn. Philadelphia, WB Saunders.2008; .pp.2708-14. 
4. New Zealand Dermatological Society Incorporated 5. eMedicine Specialties $>$ Dermatology $>$ Pediatric Diseases

5. Lamellar Ichthyosis : Author: Heather Kiraly Orkwis, Philadelphia College of Osteopathic Medicine Coauthor(s): Theresa Dressler Conologue, DO, FAAD, Physician, Department of Dermatology, Geisinger Medical Center; Jeffrey Meffert, MD, Assistant Clinical Professor of Dermatology, University of Texas Health Science Center-San Antonio Contributor Information and Disclosures Updated: Mar 23, 2009

6. Akiyama M, Shimizu H. An update on molecular aspects of the non-syndromic ichthyoses. Experimental Dermatology [serial online]. March 13, 2008; 17:373-382. Available from: Medline. Accessed January 17, 2009. [Medline]. Available at http:// onlinelibrary.wiley.com/doi/10.1111/j.16000625. 2007. 00691.x/pdf.

7. Bébé Collodion syndrome.www.WD.com Last Update: 26 January, 2011

8. Lane EB, Mclean WH. Keratins and skin disorders. J Pathol2004; 204: 355-66.

9. Rudrappa S, Shambulingappa B, Kumar H V. Collodion baby. Asian J Ped Pract 2005; 8: 54-56.

10 Gysel D V, Linjen RL, Moeki SS et al. Colloidion baby: a follow up of 17 cases. J European Acad Dermatol Venereal 2002; 16:472-75.
11. Pongprasit P. Collodion baby: the outcome of long term follow up. Journal Med Assoc Thai 1993; 76:17-22.

12. Sarkar R, Patwari AK, Dutta AK, et al. An appraisal of Pediatric dermatologic emergencies. IndianPediatr 2000: 37; 425-29.

13. Verma A, Uttarmani N. Colliodion baby (images in clinical practice). Indian Pediat 2001: 38: 1428

14. Frenk E. A spontaneously healing collodion baby: A light and electron microscopical study. Acta Derm Venerol 1981; 61; 168-71.

15. Harting $\mathrm{M}$, Brunetti-Pierri $\mathrm{N}$, Chan $\mathrm{CS}$ et al. Selfhealing collodion membrane and mild nonbullous congenital ichthyosiform erythroderma due to 2 novel mutations in the ALOX12B gene. Arch Dermatol 2008; 144: 351-56.

16. Shwayder T, Akland T. Neonatal skin barrier: structure, function \& disorders. Dermatol Ther 2005; 18: 87-103.

17. James, William; Berger, Timothy; Elston, Dirk (2005). Andrews' Diseases of the Skin: Clinical Dermatology. (10th ed.). Saunders. ISBN 0-72162921-0

18. Cox HC, Hinz MD, Lubno MA,et al. Clinical application of nursing diagnosis, $3^{\text {rd }}$ Edition. Philadelphia: F.A.Davis company; 1997, p.93-206 